\title{
Stimulus range, number of categories, and the "virtual" exponent
}

\author{
HUGH J. FOLEY, DAVID V. CROSS, MARY ANN FOLEY, and RICHARD REEDER \\ State University of New York, Stony Brook, New York
}

\begin{abstract}
Three different stimulus modalities (line length, number, and sound pressure) were judged by magnitude scaling techniques and by 7-, 15-, 31-, and 75-point category scales. All of the 40 subjects were given the same number stimuli, but two different sound-pressure ranges were presented (each to 20 subjects) and four different line-length ranges were presented (each to 10 subjects). Analyses of lack of fit for various simple functions were performed to determine bestfitting functions. The simple power function was often found to be an adequate fit to the data for all the response modalities used, although all of the response modalities were sensitive to changes in stimulus range. For simple power functions, the category-8cale exponent was a function of both the range of stimuli and the number of categories provided. Category scales did not always produce exponents smaller than those obtained with magnitude estimation, which calls into question the concept of a virtual exponent for category scales.
\end{abstract}

Numerous methodologies have been developed to enable the social or behavioral scientist to explore and quantify subjects' perceptions of various stimuli. Categorical-rating scales and magnitude scales are two approaches that have been used to investigate a wide variety of physical (e.g., loudness, brightness) and social (e.g., occupational prestige, acceptability of political candidates) stimuli. However, since the results obtained when the two methods are applied to the same stimuli are often at odds, there has been a great deal of controversy over the relative merits of the two methods (e.g., Anderson, 1974a, 1974b; Marks, 1974; Stevens, 1975).

At the root of this controversy seems to be the lack of adequate models relating each of the scaling methods to the target stimuli. Were these models fully specified, then the relationship between category scales and magnitude scales could also be determined, and the controversy should evaporate. This paper attempts to move toward this goal by identifying some additional parameters that are necessary to any model relating category scales to physical stimuli.

Part of the problem facing investigators is the difficulty of evaluating alternative models by examining their goodness of fit. We are in basic agreement with Birnbaum $(1973,1974)$ that the correlation coefficient is not appropriate for this purpose, and yet researchers

We would like to acknowledge the helpful criticisms of earlier versions of this paper by D. Emmerich, G. Lockhead, J. Tanenhaus, M. Teghtsoonian, and an anonymous reviewer. The research was supported in part by Grant S041125 from the National Science Foundation. M. A. Foley is now at Nazareth College of Rochester, Rochester, New York 14610. Requests for reprints should be sent to either H. Foley or D. Cross, Department of Psychology, S.U.N.Y. at Stony Brook, Stony Brook, New York 11794. have relied on it as a measure of the goodness of fit of particular linear models. In this paper, we will be relying upon a different curve-fitting approach, one detailed by Draper and Smith (1966). ${ }^{1}$ They suggest testing the composite of the nonlinear components of the data as an index of lack of fit. If these nonlinear components are explaining a significant amount of the variability in the data, then the model is rejected even if the linear component is also significant.

First, we will briefly examine some of the studies that have attempted to characterize the relationship between category scales and magnitude scales, and the relationship between category scales and physical continua. Then we will present our own data, which were analyzed for lack of fit to four simple modelslinear, logarithmic, power, and exponential functions.

\section{What Is the Relationship Between Category}

Scales and Magnitude Scales?

The relationship between magnitude and category scales has been a topic of frequent investigation since the seminal paper of Stevens and Galanter (1957). The results of this early study and a number of experiments by different investigators suggested that the relationship between category and magnitude scales typically fell between a logarithmic and a power function (Shinn, 1974). By the use of corrective constants, it was possible to adjust the relationship between the two scales so that it was more nearly a logarithmic function (Eisler, 1962b; Galanter \& Messick, 1961) or more nearly a power function (Marks, 1968; Stevens, 1975). Because the constants were not attributed to the effects of empirical variables, such approaches are far from satisfying, however appropriate they may be from a mathematical or measurement perspective.

Attempts to reconcile the differences between these 
two scaling procedures have typically focused upon the range of stimuli presented and the range of responses (i.e., the number of categories in the category scaling procedure) used by the subject (Marks, 1968; Montgomery, 1975; Stevens \& Galanter, 1957). Stevens and Galanter (1957) concluded that the number of categories offered the subject had no effect on the relationship between category and magnitude scales. Marks (1968), however, concluded that the number of categories might have some effect on the relationship observed between the category scale and the stimuli being judged. Therefore, it seems reasonable to expect that the relationship between category and magnitude scales would also be affected by the number of response categories provided the subject. From a more general perspective, Montgomery (1975) argued that the constraints upon the subject's responses produced the nonlinear relationship often observed in relating category and magnitude scales. When comparing subjects who were forced to respond with a wide and open response modality with those who were forced to respond with a narrow and closed response modality, Montgomery found a curvilinear function like that typically found to relate categorical and magnitude responses.

Since the number of response categories in a rating scale is established by the experimenter and not by the subject, it is likely that the differences in results between the two procedures could be eliminated by the appropriate selection of the number of categories offered to the subject (Gibson \& Tomko, 1972). This seems especially reasonable when we consider that the subject is typically free to use any response range in a magnitude estimation experiment.

\section{What Is the Best Function Relating Category Scales and Physical Stimuli?}

In addition to the question of the relationship between category and magnitude scales, researchers have also been interested in the form of the function relating category scales and physical stimuli. Some evidence in the literature suggests that the relationship between category scales and physical stimuli is a simple logarithmic function (Galanter \& Messick, 1961; McKelvie, 1978; Montgomery, 1975). Other evidence, however, points to the power function as best describing the relationship between categorical responses and physical stimuli (Eisler, 1962a, 1963; Montgomery, 1975).

Because of the wide variation in stimulus sequences and ranges, and other methodological factors (e.g., whether anchoring stimuli are used or not, the type of rating scale, whether the subject is shown the full range of stimuli in advance or not) in these various studies, it is impossible to reconcile the conflicting findings reported. Furthermore, one would not want to rely upon the relatively small difference in correlation coefficients typically found as a criterion to determine the best functional fit for categorical data.
Probably the best known study in which it is argued that the relationship between category scales and physical stimuli is a power function (but with an additional parameter inserted) is that of Marks (1968). Marks used three different ranges $(15,30$, and $45 \mathrm{~dB})$ of six luminance stimuli and three different category scales (4-, 20-, and 100-point). As is often the case, subjects' categorical responses were made with numbers (i.e., the subject was instructed to give the number " 1 " in response to the smallest stimulus, and the number " 4 ," " 20 ," or " 100 " to the largest stimulus).

Marks chose to fit the following formula to the data:

$$
\log \left(\mathrm{C}+\mathrm{C}^{\prime}\right)=\alpha \log \mathrm{S}+\log \mathrm{a},
$$

where $\mathrm{C}$ is the categorical response, $\mathrm{C}^{\prime}$ is determined by an iterative procedure to maximize the correlation coefficient, $\alpha$ is the exponent, $\mathrm{S}$ is the stimulus level, and $\mathrm{a}$ is a constant of proportionality.

After estimating data points from Marks's Figure 1, it was determined that the correlations for the powerfunction fits (with three parameters) are marginally better than, or equivalent to, the logarithmic fits (with two parameters). These differences are more marked with the larger stimulus ranges, but all of the correlations for both functions are greater than .97 . Thus, the inclusion of the constant has not been given empirical explanation, it makes the model less parsimonious, and it still does not establish the power function as the superior explanation of the data even after the adjustment.

As an examination of the values of $C^{\prime}$ derived by Marks (and summarized in Table 1) shows, the constant is anything but constant, and actually increases systematically as the number of categories increases and as the range of the stimuli decreases. Furthermore, as the log-range of response $\left(\log R_{\psi}\right)$ for the unadjusted responses and the log-range of response after the correction show, the additive constant serves to compress the range of categorical response, with the unavoidable effect of lowering the obtained (virtual) exponent. The average exponent computed on the adjusted data is .13, which is well below the exponent .33 typically observed in magnitude estimation experiments with this stimulus modality, but the average exponent for the unadjusted data is .35-or approximately the same as one would obtain with magnitude estimation procedures. On the basis of the experiments Marks reported, it is clear that the discovery of a "virtual" exponent (i.e., one much less, and often half that obtained by magnitude estimation techniques) is dependent upon the addition of $\mathrm{C}^{\prime}$ to the model. It seems that $\mathbf{C}^{\prime}$ is a parameter that models the joint effect of the stimulus range and number of response categories. If $\mathrm{C}^{\prime}$ is left out of the model, these effects are exhibited in the obtained exponent to a far greater degree.

Marks (1968) also summarized 20 experiments on 
Table 1

Summary Data From Marks (1968)

\begin{tabular}{|c|c|c|c|c|c|c|c|c|c|c|}
\hline \multirow{2}{*}{$\begin{array}{l}\text { Number of } \\
\text { Categories }\end{array}$} & \multirow[b]{2}{*}{ Measure } & \multicolumn{9}{|c|}{ Stimulus Range (dB) } \\
\hline & & & 15 & & & 30 & & & 45 & \\
\hline 4 & $\begin{array}{l}\mathbf{C}^{\prime} \\
\log \mathbf{R}_{\psi} \\
\log \mathbf{R}_{\psi^{\prime}}\end{array}$ & 10.5 & .48 & .08 & 3.25 & .54 & .21 & 0.1 & .60 & .57 \\
\hline 20 & $\begin{array}{l}\mathbf{C}^{\prime} \\
\log \mathbf{R}_{\psi} \\
\log \mathbf{R}_{\psi^{\prime}}\end{array}$ & 27.0 & .65 & .16 & 8.8 & 1.24 & .43 & 4.7 & 1.27 & .61 \\
\hline 100 & $\begin{array}{l}\mathbf{C}^{\prime} \\
\log \mathbf{R}_{\psi} \\
\log \mathbf{R}_{\psi^{\prime}}\end{array}$ & 185.0 & .67 & .13 & 25.0 & 1.65 & .63 & 10.4 & 1.97 & .96 \\
\hline
\end{tabular}

Note-The unadjusted response range is $R_{\psi}$, and the adjusted response range is $R_{\psi}$.

categorical-rating procedures performed by other researchers. An examination of his Table 1 indicated that the data were often fit relatively well by power functions without adding $\mathrm{C}^{\prime}$, since, in 12 of the 20 experiments, $\mathbf{C}^{\prime}$ averaged less than one category unit (mean = $.659, \mathrm{SD}=.40$ ). Again, given that a 7-point category scale is typically used in these studies, it is not surprising to find that the exponents for these experiments are less than those one would obtain in magnitude estimation experiments. With category scales containing fewer than 31 points, one would predict power functions with exponents less than those typically obtained by magnitude estimation techniques, given Teghtsoonian's (1971, 1973) research, since $\log R_{\psi}$ for magnitude estimation is 1.53 and $\log \mathbf{R}_{\psi}$ for category scales of 31 points or fewer would be 1.49 at most. Teghtsoonian showed that the exponent for a modality can be approximated by the ratio of the logarithmic response range $\left(\log R_{\psi}\right)$ over the logarithmic stimulus range $\left(\log R_{\phi}\right)$. Since the response range was fairly constant across stimulus modalities, changes in the exponent could be predicted by the differences in the stimulus range. Where stimulus range is constant, one would predict decreasing exponents when the response range is attenuated. Forcing subjects to respond with numbers between 1 and 20 should produce a lower exponent than forcing them to respond with numbers between 1 and 100 to judge the same stimulus range. Furthermore, because the additive constant serves to lessen the log range of responses for a particular scale, it is not surprising that even smaller exponents were obtained by Marks in the analysis of his own data or those of others.

It may be that the power function (possibly with the inclusion of some empirically based parameters to model stimulus range, number of stimuli, etc.) is an adequate model for the relationship between category scales and physical stimuli, but the notion of a "virtual" exponent as described by Marks (1968) and Stevens (1975) does not seem to be adequately justified.

\section{Overview of the Current Research}

The present study was designed to investigate two aspects of category scales. First, we were interested in examining the relationship between category and magnitude scales matched to the same stimuli to see the extent to which a concave curvilinear function is found to relate the two scales. Second, we were interested in providing further evidence as to the form of the simplest function relating category scales to physical stimuli by testing the adequacy of simple linear models for the relationship. Further, if a simple power function does provide a reasonable fit, we were interested in examining whether the exponent relating category scales to physical stimuli (the virtual exponent) was different from that relating magnitude scales to physical stimuli, as suggested by Marks $(1968,1974)$ and Stevens $(1971,1975)$.

In order to pursue these interests, we used both a wider range of categorical responses than typically used (7-, 15-, 31-, and 75-point category scales) and magnitude responses (line production and magnitude estimation) to obtain judgments of stimuli presented in different stimulus modalities, which themselves varied in range across groups of subjects.

\section{METHOD}

\section{Subjects}

Forty undergraduates from the State University of New York at Stony Brook volunteered to participate in this study. Four of the subjects had participated in previous psychophysical scaling tasks, but were not aware of the purposes of this study.

\section{Stimulus Modalities}

There were three stimulus modalities included in this studyline length, number, and sound pressure. There were 10 levels within each modality, and each level was presented six times within the 60 trials for a particular stimulus modality. The subject was presented with one of three random orders of the 60 stimulus presentations for any modality.

All 40 subjects saw the same number stimuli, which covered a range of 144:1. The stimulus levels were $1,2,3,5,9,16,27$, 48,83 , and 144. For the sound-pressure stimulus modality, there were two ranges: one of $25 \mathrm{~dB}$ SPL $(60,62,65,67,70$, $72,75,77,80$, and $85 \mathrm{~dB})$ and one of $45 \mathrm{~dB}$ SPL $(55,60,65$, $70,75,80,85,90,95$, and $100 \mathrm{~dB}$ ). Twenty of the subjects were presented with the narrower range, and the other 20 were presented with the wider range. For the line-length-stimulus modality, 10 different subjects judged each of four different ranges-7.5:1 $(2,2.5,3,4,5,6,7,9,11$, and $15 \mathrm{~cm}), 15: 1(2.5$, $3,5,7,10,15,18,22,31$, and $38 \mathrm{~cm}), 31: 1(1,1.5,2,3,5,7$, 
$10,15,22$, and $31 \mathrm{~cm})$, and $72: 1(.5,1,1.5,2,3,5,9,15,22$, and $36 \mathrm{~cm}$ ).

Stimulus presentation was controlled by a Digital Equipment Corporation PDP-12A computer with a point-plot display (VR-14). A television camera (Sony AVC-32000) was fixed in position in front of the point-plot display, and the image was transmitted to a television monitor in a separate sound-attenuated subject room.

White line and number stimuli were presented against a black background on the television monitor, which was located approximately $4 \mathrm{ft}$ in front of the subject. The sound-pressure stimuli were 1-sec bursts of white noise produced by a GrasonStadler white-noise generator, and the levels of the noise were controlled by the computer through an attenuator programmable in .5-dB steps. The noises were presented binaurally and in phase through headphones (TDH-39).

The order in which a particular subject was presented with the stimulus modalities was randomized. Before each stimulus modality was presented, the subject was given instructions about the particular modality to be judged, and then the stimulus levels were presented briefly to familiarize the subject with the range.

\section{Response Modalities}

Each stimulus was judged with six different response modalities. Within each of the three stimulus modalities, each stimulus was judged by a 7-, 15-, 31-, and 75-point category scale. In addition, number stimuli were judged by line length and soundpressure responses, sound-pressure stimuli were judged by line length and number responses, and line-length stimuli were judged by sound pressure and number responses.

Before each block of 60 trials for a given stimulus modality, the subject was given a packet of 10 pages $(21.5 \times 35.5 \mathrm{~cm})$, with a space for six responses on each page. The packet was positioned lengthwise on the table in front of the subject. All responses were made in this packet, with the exception of the sound-pressure responses, and on each page the responses that were recorded were all from different response modalities.

\section{Procedure}

On each trial, the subject saw the stimulus presented in the middle of the television screen (e.g., a line or a number value) or the word "NOISE" to indicate that the particular trial included the presentation of a sound-pressure level. The trial number and the type of response required on that trial were also displayed on the television monitor at the bottom of the screen. The stimulus presentation rate was controlled by the subject, who pressed a button to indicate that a response had been made, after which the next stimulus was presented. All 60 trials for one stimulus modality (e.g., numbers) were presented before the subject received any stimulus presentations from the other modalities, and before receiving further instruction concerning responses to those other modalities.

At the beginning of the session, the subject was told that the purpose of the study was to measure the magnitude of perceptions, and that the subject would be asked to report those magnitudes in a number of ways. The subject was shown the four types of category scales that would be used, and the subject was given four practice stimuli from two modalities. (These practice stimuli were not included among the stimuli to be jidged later.) After being shown the full range of sound-pressure responses ( 40 to $100 \mathrm{~dB}$ SPL), the subject attempted to match three randomly selected sound-pressure settings. The subject was then familiarized with matching numbers to the practice line lengths, and line lengths to the practice numbers. Finally, the subject matched sound-pressure responses to the practice stimuli (four line lengths and four number stimuli).

For the categorical responses, the subject was told to think of the dashes as partitioning the space between the largest stimulus in the set (which would be placed in the rightmost dash) and the smallest stimulus in the set (which would be placed in the leftmost category) into equal intervals. Thus, a stimulus that seemed to be midway between the two extreme stimuli would be placed in the middle category. The categorical responses were made by placing a mark above one of the string of horizontal dashes in the response packet, varying from 7 to 75 dashes. This procedure represents a potentially important difference between this study and other studies, in which subjects were told to use whole numbers between 1 and 7 for their responses (e.g., Marks, 1968; Stevens \& Galanter, 1957). By having no numbers associated with categories at all in the present study, the possibility of the subject's associating particular numbers with particular categories was minimized.

For the magnitude responses, no standard or modulus was assigned. The subject was told to give, to the first stimulus presented, any response that seemed reasonable, and, to subsequent stimuli, a proportionally larger response if the stimulus was larger or a proportionally smaller response if it was smaller.

Sound-pressure responses were made by means of a sone potentiometer (Beckman Helipot NL 5711-600) located on the table in front of the subject. The subject heard the series of $1-\mathrm{sec}$ bursts of noise over the headphones only when a soundpressure response was required, and the knob on the potentiometer was reset after each response so that the subject began the adjustment on the next noise trial from the lowest soundpressure level available. Sound-pressure responses were converted to digital form and stored by the computer on its magnetictape-storage facility.

Number and line length responses were made by writing numbers or drawing lines free-hand in the spaces provided in the response packet. The subjects were advised that if they needed to make a longer line-length response than the paper provided, they could draw more than one line. Most subjects drew only one line as a response. ${ }^{2}$

\section{RESULTS}

\section{Magnitude Responses}

Figures 1 and 2 show the results of the matches of the magnitude response continua to the different physical stimulus modalities. Figure 1 displays the geometric means of the number responses to the four different linelength ranges and of the line-length responses to the number stimuli. It can be seen that the fit is quite good in each case, and that the slopes of the functions are affected by the varying ranges. As is often reported, the shorter ranges have steeper slopes than the longer ranges.

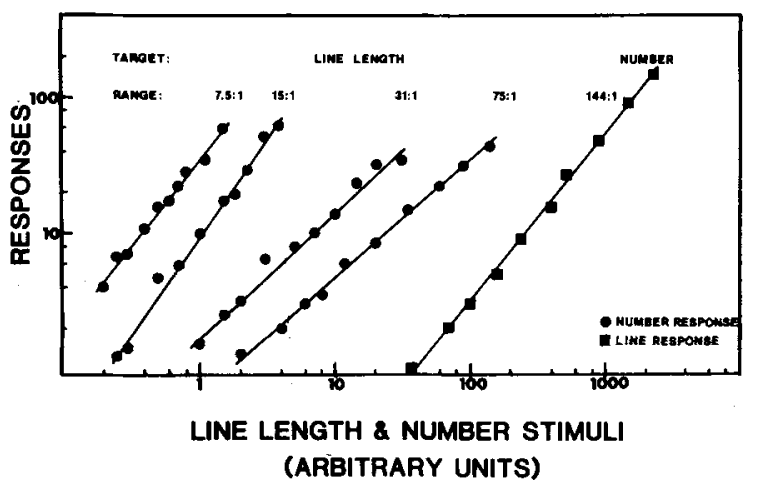

Figure 1. Geometric mean number responses to four linelength ranges and geometric mean line-length responses to number stimuli, displayed in log-log coordinates. The values on the abscissa have been arbitrarily displaced. 


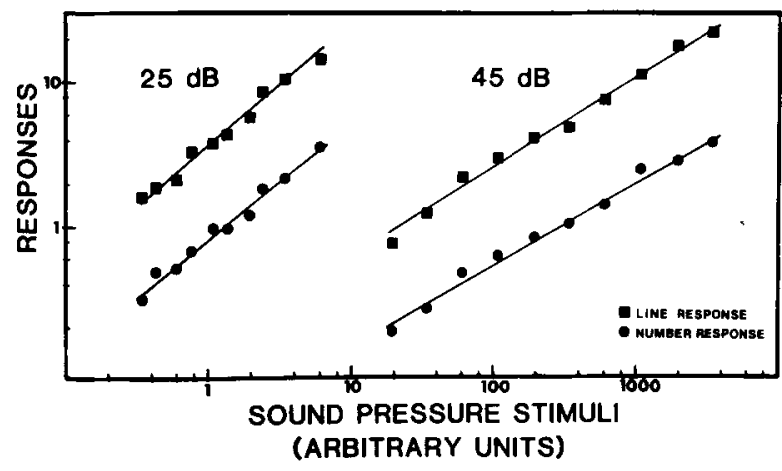

Figure 2. Geometric mean number and line-length responses to two sound-pressure stimulus ranges, displayed in $\log -\log$ coordinates. The values on the abscissa have been arbitrarily displaced.

Due to technical problems, sound-pressure responses are not included in these analyses. ${ }^{3}$

Figure 2 shows the relationship between the geometric means of the number responses and the two sound-pressure ranges, and the geometric means of the line-length responses to the same two sound-pressure ranges. Again, as is clear in these figures, the effect of stimulus range is present, with a trend toward decreasing exponent with increasing range.

Tests for lack of fit (Draper \& Smith, 1966) were performed to determine if there were trends in addition to the linear model being evaluated. ${ }^{4}$ If so, then the model being evaluated can be rejected. In the present data, the appropriateness of four functions-linear, logarithmic, power, and exponential-was evaluated. In only one instance could the power function be rejected because of significant lack of fit. For the line-length responses to the 45-dB sound-pressure stimuli, all four functions, including the power function, were rejected. Thus, there was no instance in which the simple power function was rejected and another model was not.

As a result of the exclusion of the sound-pressure response data, the only indirect cross-modality matching functions that could be examined were the two for the sound-pressure stimuli. Figure 3 shows these indirect matches of line length to number responses via the two sound-pressure ranges. With exponents of 1.0 and 1.1 (for the narrow and wide stimulus ranges, respectively), neither of the exponents is significantly different from the predicted slope of 1.0 , nor does the difference in the stimulus range seem to produce a major change in the functions. This suggests that stimulus range affects the different response modalities equally, at least for these modalities.

Given that different stimulus ranges were used in this study, we were also interested in comparing our results with those obtained by Teghtsoonian (1973). Figure 4 shows the exponents from our experiment, as well as some of the exponents from Teghtsoonian (1973, Figure 4). The intramodal range effects in this study are greater than one would expect from the relatively flat functions reported by Teghtsoonian. Even if one were to exclude the exponent for the 15:1 line-length range (discussed below), the intramodal range effects remain steeper than expected.

In attempting to explain a seemingly aberrant exponent for the number matches to the 15:1 line-length range, a potentially important factor emerged that might

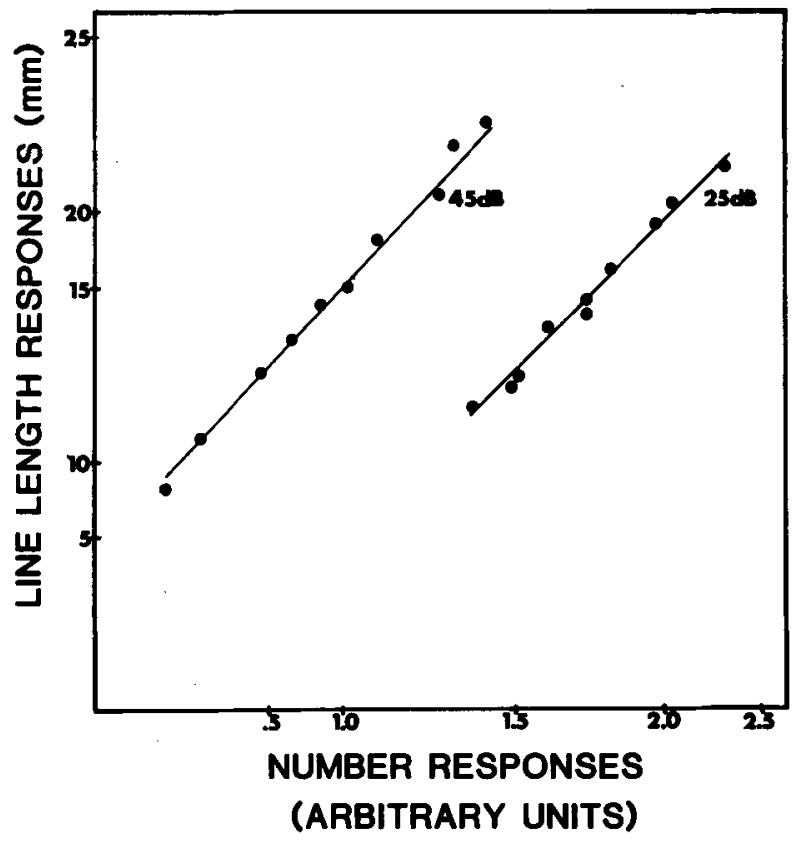

Figure 3. Indirect cross-modality matching functions for linelength and number responses to two sound-pressure stimulus ranges, displayed in $\log -\log$ coordinates.

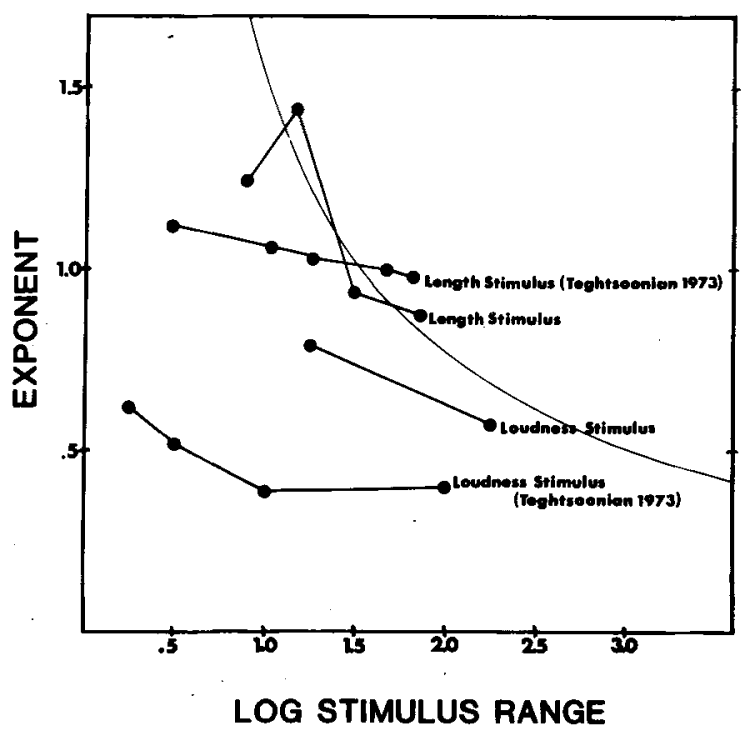

Figure 4. Exponents for magnitude estimation as a function of logarithmic stimulus range $\left(\log R_{\phi}\right)$ from Teghtsoonian (1973) and the current study. 
operate on the value of the exponent-namely, the adaptation level. Because the stimulus series were selected only on the basis of range from smallest to largest stimulus, no consideration was given to the "average" of the series. As it turns out, the geometric means of the four line-length ranges, in increasing order of range, are $5.3,10.6,5.6$, and $4.3 \mathrm{~cm}$. Thus, the geometric mean of the 15:1 stimulus range was about twice that of the other series, and, contrary to expectations, the exponent obtained by magnitude estimation of these stimuli was higher than that from the $7: 1$ stimulus range. Further research will be necessary to determine how the position of the stimulus range within the dynamic range of a modality affects the exponent.

\section{Categorical Responses}

In order to assess whether the simple power function or the simple logarithmic function provided the best fit to the category scale data, the same lack-of-fit analysis performed on the magnitude response data was performed here. The picture that emerges is not quite as clear as was the case with the magnitude scale responses. In general, the power function was the best fit, although the linear fit was often quite good. Of the 28 sets of data evaluated, there were 7 instances in which all four simple functions were rejected. Of the remaining 21 sets, none could be adequately fit by an exponential model, 19 could not be adequately fit by the logarithmic model, and 9 could not be fit adequately by a linear model. The power function was rejected in only three of

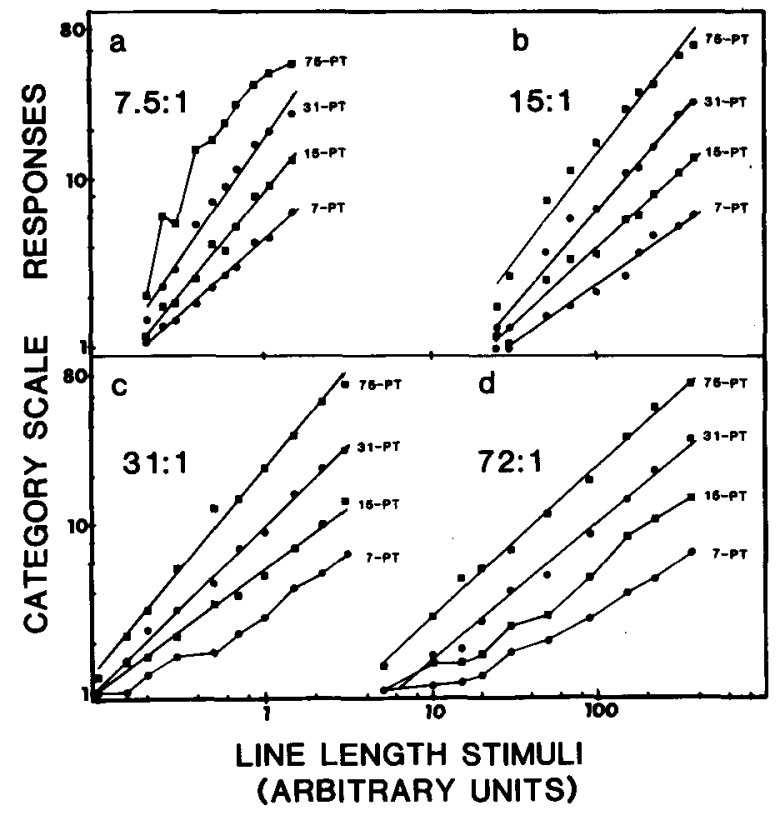

Figure 5. Geometric mean category scales matched to linelength stimuli, displayed in $\log -\log$ coordinates. Shown are the 7-, 15-, 31-, and 75-point category scales matched to the (a) $7.5: 1$, (b) $15: 1$, (c) $31: 1$, and (d) $72: 1$ line-length ranges. Straight lines indicate the line of best fit for the power function. Where there was significant lack of fit for the power function, points are simply connected.

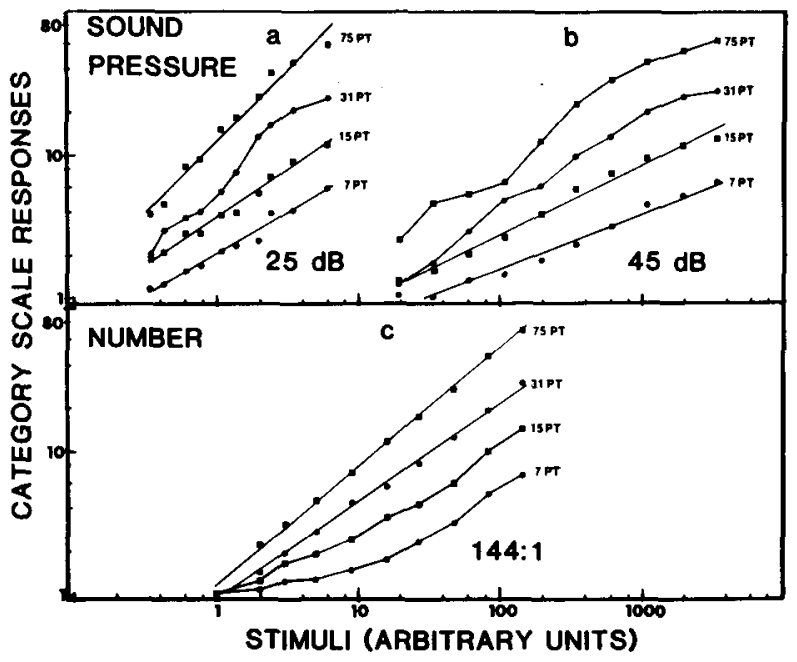

Figure 6. Geometric mean category scales matched to sound pressure and number stimuli, displayed in $\log -\log$ coordinates. Shown are the 7-, 15-, 31-, and 75-point category scales matched to the (a) 25-dB sound-pressure range, (b) 45-dB sound-pressure range, and (c) 144:1 number range. Straight lines indicate the line of best fit for the power function. Where there was significant lack of fit for the power function, points are simply connected.

these 21 sets (for a total of 10 instances out of the 28 in which the power function was not an adequate fit). The mean correlation coefficient for the rejected power functions was .98 , showing the sensitivity of the lack-offit analysis to nonlinearity. Figures 5 and 6 display the results of the power-function fits for the 28 data sets. Stevens (1975) and others (e.g., Marks, 1968, 1974; Schneider, Parker, Valenti, Farrell, \& Kanow, 1978) have argued that the relationship between category scales and stimuli is a power function, and these data bear out that position.

Figures $7 \mathrm{a}$ and $7 \mathrm{~b}$ show the exponents obtained from the power-function fits to the various stimuli. The four curves shown in the two figures are not derived empirically (as in Teghtsoonian, 1973), but instead represent the exponents that one would predict if sub. jects used the full category scale at each stimulus range (i.e., these are maximum obtainable exponents from simple power-function fits, with a $\log \mathbf{R}_{\psi}$ of .85 for the 7-point category scale, 1.18 for the 15-point category scale, 1.49 for the 31-point category scale, and 1.88 for the 75-point category scale). Thus, if all subjects placed the smallest stimulus in the lowest category and the largest stimulus in the highest category, with the other stimuli in between, the exponents would fall on the curves. To the extent that the exponents are less than predicted by the curve, the category scale is not being fully utilized, on average. Accordingly, it is not surprising that, as the stimulus range increases, the exponents begin to approach the curves. Since the $1.5 \log$ units of response range for magnitude estimation experiments are roughly equivalent to the log response range for the 31 -point category scale, it is clear that, with category 


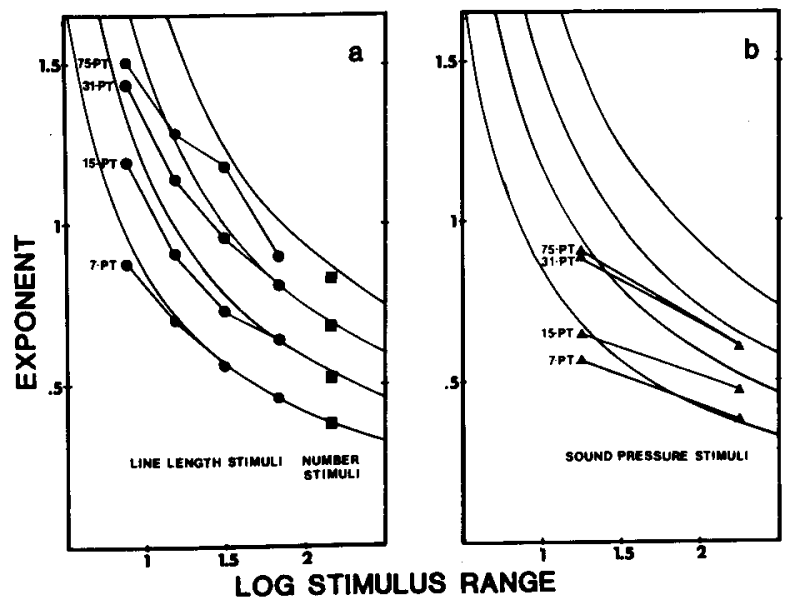

Figure 7. Category scale exponents as a function of logarithmic stimulus range $\left(\log R_{\phi}\right)$, (a) for line-length and number stimuli, and (b) for sound-pressure stimuli.

scales having fewer than 31 points, the exponent will be less than that for a magnitude estimation experiment. Also, with moderate ranges of stimuli and category scales of 31 points or more, the exponent would probably be less than that obtained in a magnitude estimation experiment, since the full scale would not be used by all subjects.

It is clear that the exponents obtained in this experiment are dependent upon both the range of the stimuli being judged and the number of categories being offered to the subject for response. Thus, as shown in Figures 7a and $7 \mathrm{~b}$, for any given category scale, the exponent decreases as the stimulus range increases, as is also the case with the magnitude response data. In addition, however, at each stimulus range the exponent decreases as the number of categories offered to the subject decreases, as one would expect simply because the maximum $\log R_{\psi}$ values are constrained by the number of categories. A comparison of Figure 7 and Figure 4 reveals that the category-scale exponents are not always half of the magnitude-scale exponents, and that with large numbers of categories the exponents for category scales can be larger.

\section{Relationships Between Category and Magnitude Scales}

Because of the fact that both categorical and magnitude responses are subject to error, it is not possible to assess the lack of fit of these relationships using a least squares approach. Given that the linear and power function fits of both the magnitude and categorical responses to these stimuli typically provided acceptable fits, both functions were fit to the interrelationship between the magnitude and category responses. The slopes for these functions were assessed using an errorin-both-variables model (Cross, 1974).

The value of the exponent relating category and magnitude scales was found to be affected more by the number of categories than by the range of stimuli.
Given the decreasing monotonic functions relating the exponents for both magnitude- and category-scale data to stimulus range, the result is predictable. The indirect cross-modality match between two magnitude scales shows a similar relationship and presumably occurs because the stimulus range affects the response modalities equally.

A strong linear component of the relationship between the category and magnitude scales was obtained, as Gibson and Tomko (1972) have also demonstrated. Thus, the curvilinear relationship so often found does not always characterize the relationship between category and magnitude scales. Montgomery (1975) has demonstrated that the curvilinearity stems from relating a relatively constained (narrow and closed) response range to a relatively unconstrained (wide and open) response range. As the number of categories is increased (wide and closed), the curvilinearity is lessened.

\section{DISCUSSION}

Two commonly accepted notions about category scales have been called into question by the results of this study. The first is that the relationship between category and magnitude scales is typified by a concave curvilinear function. The present study adds support to the findings of Gibson and Tomko (1972) and Montgomery (1975) that the form of the function relating category and magnitude scales is variable and greatly dependent upon the response ranges available to the subject. The concave curvilinear function is obtained when there is a great disparity between the range of response offered to the subject on the two response scales.

However, increasing the number of categories available to the subject in the rating scale should not be perceived as a panacea. When subjects are constrained to use a response range that is different from one they would choose in an unconstrained experiment, their results are quite different (Baird, Kreindler, \& Jones, 1971; King \& Lockhead, 1981). For instance, in the current study, the power function was rejected when the number of categories in the scale and the range of the stimuli was most discrepant (75-point category scale matched to 7:1 line-length range and 7- and 15-point category scales matched to the 75:1 line-length and 144:1 number ranges). Since the stimulus range is typically fixed by the experimenter, a relatively unconstrained response modality should be preferred.

A second commonly accepted notion that was called into question by the present study is that of the virtual exponent. Our own results and a reanalysis of Marks's (1968) data demonstrate that the category-scale exponent need not be less than the exponent from a magnitude scale matched to the same stimuli. Obtaining a power-function exponent for category scales that is less than that obtained with magnitude estimation may be an artifact of the utilization of an additive constant for rating scales with large numbers of categories, or more 
generally because category scales that have been used in the past have fewer than 31 points (typically, 7,9 , or 11). Interestingly, the common 7-point category scale would provide a $\log \mathrm{R}_{\psi}$ of .84 , or a little over half of the typical $\log R_{\psi}$ of 1.53 found for magnitude estimation (Teghtsoonian, 1973). Since this has been the most commonly used category scale, it is clear that, even without additional parameters in the power function model, one would expect the "typical" exponent for category scales to be about half that found with magnitude estimation.

This study also demonstrated that the power function was a better model in fitting these data than was the logarithmic model. Evaluation of the lack of fit of a function is a more appropriate test of a model than using the correlation coefficient as an index of goodness of fit. In instances in which the correlation coefficents are quite high, the evaluation procedure used in the present study is able to detect deviations from the model and assess their significance.

Just as the investigation of the relationship between magnitude scaling and various sensory modalities is continuing in an effort to determine the relevant parameters in the magnitude scale model, so should a similar research program be directed toward category scales. It seems reasonable that there are common contextual influences on the two scaling procedures (stimulus order, stimulus range, stimulus spacing, and adaptation level) as well as problems specific to category scales (number of categories in the scale, possibly the number of stimuli being judged). The relationship between category and magnitude scales can be understood only when the relationship of each procedure to the stimulus modality is better understood.

\section{REFERENCES}

Anderson, N. H. Algebraic models in perception. In E. C. Carterette \& M. P. Friedman (Eds.), Handbook of perception (Vol. 2). New York: Academic Press, 1974. (a)

Anderson, N. H. Information integration theory: A brief survey. In D. H. Krantz, R. C. Atkinson, R. D. Luce, \& P. Suppes (Eds.), Contemporary developments in mathematical psychology (Vol. 2). San Francisco: Freeman, 1974. (b)

Baird, J. C., Kreinder, M., \& Jone8, K. Generation of multiple ratio scales with a fixed stimulus attribute. Perception \& Psychophysics, 1971, 9, 400-403.

Brrnbaum, M. $H$. The devil rides again: Correlation as an index of fit. Psychological Bulletin, 1973, 79, 239-242.

Brrnanum, M. H. Reply to the devil's advocates: Don't confound model testing and measurement. Psychological Bulletin, 1974, 81, 854-859.

Cross, D. V. Some technical notes on psychophysical scaling. In H. Moskowitz, B. Scharf, \& J. C. Stevens (Eds.), Sensation and measurement: Papers in honor of S. S. Stevens. Dordrecht, Holland: Reidel, 1974.

Draper, N. R., \& SMtth, H. Applied regression analysis. New York: Wiley, 1966.

Eisler, H. On the problem of category scales in psychophysics. Scandinavian Journal of Psychology, 1962, 3, 81-87. (a)

Eister, H. Empirical test of a model relating magnitude and category scales. Scandinavian Journal of Psychology, 1962, 3, 88-96. (b)

Ersler, H. Magnitude scales, category scales, and Fechnerian integration. Psychological Review, 1963, 70, 243-253.
Galanter, E., \& MEssick, S. The relation between category and magnitude scales of loudness. Psychological Review, 1961, 68, 363-372.

Gibson, R. H., \& Tomко, D. L. The relation between category and magnitude estimates of tactile intensity. Perception a Psychophysics, 1972, 12, 135-138.

HAYs, W. L. Statistics for the social sciences (2nd ed.). New York: Holt, Rinehart, \& Winston, 1973.

KePpEL, G. Design and analysis: $A$ researcher's handbook. Englewood Cliffs, N.J: Prentice-Hall, 1973.

KIng, M. C., \& LockheAD, G. R. Response scales and sequential effects in judgment. Perception \& Psychophysics, 1981, 30, 599-603.

MARKs, L. E. Stimulus-range, number of categories, and form of the category scale. American Journal of Psycyhology, 1968, 81, 467-479.

Marks, L. E. On scales of sensation: Prolegomena to any future psychophysics that will come forth as science. Perception \& Psychophysics, 1974, 16, 358-376.

McKelvie, S. J. Graphic rating scales-How many categories? British Journal of Psychology, 1978, 69, 185-202.

Montgomery, H. Direct estimation: Effect of methodological factors on scale type. Scandinavian Journal of Psychology, 1975, 16, 19-29.

Schneider, B., Parker, S., Valenti, M., Farrell, G., \& KANOW, G. Response bias in category and magnitude estimation of difference and similarity for loudness and pitch. Journal of Experimental Psychology: Human Perception and Performance, 1978, 4, 483-496.

Shinn, A. M., Ja. Relations between scales. In H. M. Blalock, Jr. (Ed.), Measurement in the social sciences: Theories and strategies. Chicago: Aldine, 1974.

Stevens, S. S. Issues in psychophysical measurement. Psychological Review, 1971, 78, 426-450.

Stevens, S. S. Psychophysics: Introduction to its perceptual, neural, and social prospects. New York: Wiley, 1975.

Stevens, S. S., \& Galanter, E. H. Ratio scales and category scales for a dozen perceptual continua. Journal of Experimental Psychology, 1957, 54, 377-410.

Tegrtsoonian, R. On the exponents in Stevens' law and the constant in Ekman's law. Psychological Review, 1971, 78, 71-80.

Teghtsoonian, R. Range effects in psychophysical scaling and a revision of Stevens' law. American Journal of Psychology, 1973, 86, 3-27.

\section{NOTES}

1. Draper and Smith (1966) and others (Hays, 1973, pp. 680684 ; Keppel, 1973, pp. 401-423) have detailed means for evaluating the degree of lack of fit of a linear model. A computer program (available from $H$. Foley on request) was written to evaluate four simple functions which could be linearized by taking logarithms of the independent and dependent variables (either one at a time or together).

2. Because of computer-memory limitations, the program could not handle stimulus presentation and all of response acquisition, thus necessitating the use of paper-and-pencil responses for category scale, line length, and number responses.

3 . Due to problems with the recording equipment, smaller sound-pressure responses were lost in the noise of the system. This produced a flatter function at the low end of the curve, thereby lowering the exponents obtained relating stimuli and sound-pressure responses. For this reason, it was decided that the sound-pressure responses should be excluded from the analysis. However, it should be noted that the effects of intrastimulus range and response range parallel those found with other magnitude judgments (i.e., the exponents decreased with increases in range).

4. All hypothesis testing was done with $\alpha=.05$.

(Manuscript received December 15, 1982; revision accepted for publication June 28,1983 .) 